\title{
Hybrid Multiobjective Genetic Algorithm with a New Adaptive Local Search Process
}

\author{
Salem F. Adra \\ Department of Automatic Control \\ \& Systems Engineering \\ University of Sheffield, Sheffield \\ (UK) +44 1142225236 \\ S.Adra@sheffield.ac.uk
}

\author{
Ian Griffin \\ Department of Automatic Control \\ \& Systems Engineering \\ University of Sheffield, Sheffield \\ (UK) +44 1142225682 \\ I.Griffin@sheffield.ac.uk
}

\author{
Peter J. Fleming \\ Department of Automatic Control \\ \& Systems Engineering \\ University of Sheffield, Sheffield \\ (UK) +44 1142225663 \\ P.Fleming@sheffield.ac.uk
}

\begin{abstract}
This paper is concerned with a specific brand of evolutionary algorithms: Memetic algorithms. A new local search technique with an adaptive neighborhood setting process is introduced and assessed against a set of test functions presenting different challenges. Two performance criteria were assessed: the convergence of the achieved results towards the true Pareto fronts and their distribution.
\end{abstract}

\section{Categories and Subject Descriptors}

I.2.8 [Computing Methodologies]: Artificial Intelligence problem solving, control methods, and search - heuristics methods.

\section{General Terms}

Algorithms, Performance, Experimentation, Theory.

\section{Keywords}

Memetic algorithms; local search; multiobjective optimization.

\section{INTRODUCTION}

Memetic algorithms are increasingly thriving metaheuristics for solving multiobjective optimization problems. These hybrid algorithms have been applied to a wide variety of problems such as image segmentation and radiotherapy treatment planning. They have proved to be highly effective, outperforming similar approaches such as pure evolutionary algorithms or stand-alone local search techniques in several application domains in terms of convergence towards Pareto-optimal solutions. While genetic algorithms are good at coarse search of the entire solution space, due to their population-based approach, they are not well suited for fine-tuning structures that are already close to optimal.

In this paper, a new local search technique is introduced and hybridized with a multiobjective genetic algorithm (MOGA) [1] The new hybrid algorithm is then tested against a well-established set of optimization problems of varying complexity. These problems feature typical Pareto front surfaces that multiobjective optimizers frequently encounter [3]. Conventional questions

Copyright is held by the author/owner(s).

GECCO'05, June 25-29, 2005, Washington, DC, USA.

ACM 1-59593-010-8/05/0006 usually raised in the field of hybrid evolutionary algorithms were addressed and answers were postulated based upon the optimization results produced. These questions include: Where shall a local search process be hybridized with a genetic algorithm? Which individuals should be fine-tuned? And when shall the local refinement be applied?

\section{Experimentations}

At the start of this research three different local search techniques were hybridized with the MOGA: simulated annealing, hillclimbing and tabu search. The three corresponding hybrid algorithms were applied to the ZDT problems [3]. The results achieved by the hybrid algorithms were compared with the results achieved by the standard MOGA. In order to provide a well-based comparison, these algorithms had to be balanced in terms of the number of objective function evaluations that were performed.

\section{Experimental Observations}

The hybrid "MOGA/Hill climbing local search" algorithm was more suitable for the ZDT problems and was outperforming the standalone MOGA and the MOGA hybridized with the other local search techniques. Despite their well recognized benefits, the extra functionality of the simulated annealing (i.e. escaping local optima) and the tabu search (keeping record of previously visited solutions) were unnecessary adds-ons in the context of most of these problems and consequently redundant computational effort was allocated to these local search functionality at the expense of the evolutionary global search. It was further noted that finetuning either all the individuals of the genetic population or just the dominated individuals was similarly effective. Either approach was significantly better than applying the local search process to just the non-dominated solutions in terms of convergence towards and spread along the Pareto front. It was notable that the process of fine-tuning the non-dominated individuals resulted in unwanted genetic drift and premature convergence. Note that due to the stochastic nature of the evolutionary strategies, a well-based judgment concerning the performance of a specific algorithm can not be stated unless the whole optimization process is repeated a number of times.

After having experimented with hybridizing traditional local search techniques with a Multi Objective Evolutionary Algorithm (MOEA), it was clear that none of these techniques was dedicated for solutions' distribution enhancement, an important performance criterion of optimization techniques. The local search introduced in this work is devoted to fine tuning the solutions 
achieved by a MOEA while committing to enhancing the solutions' distribution.

\section{Performance and Testing Techniques}

In this study two known performance indicators were employed: the "Generational distance" metric [2] [3], which measures the proximity of every non-dominated solution to the closest part of the Pareto front. The second indicator used in this study was the "Spread metric", which measures the solutions' diversity [3]. The performance of the algorithm is then articulated in term of the resulting distributions of generational distance and spread. A statistical comparison of two configurations is then possible through the use of a test statistic. In this study, the mean difference between two generational distance and spread distributions are taken as the test statistic. The importance of the experiential result is then assessed using randomization testing.

\section{The Hybrid Algorithm}

The hybridization interface between the newly implemented local search and MOGA is located prior to the selection for recombination step. This choice of location is designed to make any beneficial effects of the local search operator available to the genetic selection and recombination process.

\subsection{The Adaptive Neighborhood Setting}

The local search refinement step receives its input, in the form of the whole population of solutions from the global search process of the genetic algorithm. The local search then adaptively determines the size of the neighborhood for each individual in the population, and more precisely the neighborhood range for each decision variable that constitutes each individual. This adaptive mechanism of setting the size of the neighborhood is directly linked to the spread of the current individuals in the population. At every iteration of the local search, the current spread metric is calculated and used in the sigmoid function to determine a number in the range [0 1 1]. This denotes a percentage of the global search range for each decision variable. When the spread of the solutions is damaged due to effects such as genetic drifts and premature convergence, the local search process extends the size of the current solutions' neighborhood, thereby extending the search range in order to explore solutions in a wider neighborhood. The process is reversed when the distributions of the solutions is good in order to fine tune the solutions in a more tightly constrained neighborhood without detriment to the spread of solutions

\subsection{Perturbing the Population}

After the adaptive setting of the neighborhood, the local search process moves the current population of solutions to another place in the neighborhood by perturbing stochastically a certain number of decision variables constituting each individual. The nondominated solutions are then extracted from the perturbed and the original population of solutions and kept in an updatable online archive. The actual fitness of each individual in the current population is then compared with the fitness of its peer individual from the perturbed population, and only the fittest is kept in the current population passed to the next iteration of the local search.

At the final iteration of the local search, the population of nondominated individuals might end up being over or under populated compared to the fixed size of the population. When the population is under populated (i.e. contains less individuals then the fixed population size) at the last iteration of the local search, the gap is filled with the remaining dominated individuals extracted from the perturbed and the original populations of the last few iterations, sorted based on their exact fitness. In other words, the fittest dominated individuals are reinserted into the final population of the local search in order to maintain the original size of the population. On the other hand, when the final population is over populated, a sorting process is applied on that population based on the fitness of the individuals and the population size is reduced to the original size by excluding the worst non-dominated solutions.

\section{Results and Conclusions}

Compared to the traditional results achieved by the MOGA, the experimental results produced by the Memetic algorithm have demonstrated an improved optimization performance for some of the ZDT test functions and have demonstrated that an optimization strategy might cope well with some features and might not be well tuned to deal with other features such as the shape of Pareto fronts. The hybrid algorithm has ensured to the decision maker enhanced objectives values previously undetected by the MOGA, reflecting better fitness values for some of the ZDT functions. On the other hand it was very obvious that although the MOGA and the hybrid algorithm were balanced in term of objective function evaluation all along the experimentations, the hybrid optimizer was much faster to achieve its results compared to the computational time needed by the MOGA, a characteristic well appreciated, especially when dealing with real world applications.

\section{REFERENCES}

[1] Fonseca, C. M., Fleming, P. J.: Genetic algorithms for multiobjective optimization: Formulation, discussion and generalization, in S. Forrest (ed.), in Proceedings of the Fifth International Conference on Genetic Algorithms, Morgan Kauffman Publishers, California, pp. 416-423 1993.

[2] Purshouse, R. C.: On the Evolutionary Optimisation of Many Objectives, $\mathrm{PhD}$ thesis, Department of Automatic Control and Systems Engineering, The University of Sheffield, Sheffield, UK, 2003.

[3] Zitzler, E., Deb, K., Thiele, L.: Comparison of multiobjective evolutionary algorithms: Empirical results, Evolutionary Computation 8(2), 173-195 